\title{
Contraindications and adverse effects in abdominal imaging
}

\author{
Cosmin Caraiani ${ }^{1}$, Bianca Petresc ${ }^{1}$, Yi Dong ${ }^{2}$, Christoph F. Dietrich ${ }^{3,4}$
}

${ }^{1}$ Department of Medical Imaging, "Iuliu Hatieganu” University of Medicine and Pharmacy, Cluj-Napoca, Romania,
2Department of Ultrasound, Zhongshan Hospital, Fudan University, Shanghai, China, ${ }^{3}$ Medizinische Klinik 2 ,
Caritas-Krankenhaus Bad Mergentheim, Bad Mergentheim, Germany, ${ }^{4}$ Ultrasound Department, First Affiliated Hospital
of Zhengzhou University, China

\begin{abstract}
Ultrasound (US), computed-tomography (CT) and magnetic resonance imaging (MRI) are the most frequently used imaging techniques in abdominal pathology. US plays a pivotal role in evaluating abdominal disease, sometimes being sufficient for a complete diagnosis and has virtually no contraindications. The usage of US contrast agents will add useful diagnostic information in both hepatic and non-hepatic pathology. CT has, over MRI, the advantage of being readily available. The usage of ionizing radiation is the main pitfall of CT. Allergies and contrast induced nephropathy in patients with an impaired renal function are the major risks of contrast media administration in CT. Its excellent tissue resolution makes MRI a very useful technique in abdominal pathology, the major contraindications being the presence of MRI "unsafe" implants and devices and the presence of metallic foreign bodies, particularly close to vital structures like the eyes or major vessels. Contrast administration in MRI is restricted in patients with renal insufficiency due to the risk of nephrogenic systemic fibrosis. Allergies to MRI contrast media are rare and less important compared to allergies due to CT contrast media.
\end{abstract}

Keywords: ultrasound; computed-tomography; magnetic resonance imaging; contraindications; adverse effects

\section{Introduction}

The use of medical imaging techniques for depiction and follow-up of illness has greatly expanded during the last two decades. Abdominal diseases are no exception and diagnosis, in the field of abdominal pathology, is largely dependent on imaging techniques. Ultrasound (US), computed-tomography (CT) and magnetic reso-

Received 29.05.2019 Accepted 27.07.2019

Med Ultrason

2019, Vol. 21, No 4, 456-463

Corresponding author: Prof. Dr. med. Christoph F. Dietrich Medizinische Klinik 2, Caritas Krankenhaus Bad Mergentheim, Uhlandstr. 7, D-97980 Bad Mergentheim, Germany Phone: 49 (0)7931 - 58 - $2201 / 2200$

Fax: 49 (0)7931 - 58 - 2290

E-mail: Christoph.dietrich@ckbm.de nance imaging (MRI) have wide indications in abdominal pathology. Unfortunately, imaging techniques such as CT or MRI can produce side effects which are harmful to the patient or have contraindications which limit their diagnostic capacity [1]. The aim of this review is to summarize the contraindications and adverse effects of imaging techniques, which sometimes limit their usage.

The same collective of authors have recently published a paper "Reasons for inadequate or incomplete imaging techniques" [1]. The main idea for both papers was that, despite the developments made in the imaging field over the last decades which offer great improvements in the quality of diagnosis of abdominal pathology, there are some limitations and situations when one of the techniques is not suitable or does not offer the desired amount of information so much so that it should be replaced by another method. Contraindications for dif- 
ferent techniques and precautions that have to be taken in situations such as exposure to ionizing radiation and administration of contrast media can further diminish the amount of diagnostic information offered by imaging.

\section{Ultrasound}

US is a very useful and well-established tool for abdominal imaging. US is readily available, comparatively inexpensive, and easily accepted by the patient [2]. In most cases it is the first imaging technique used in abdominal pathology and, in many cases US offers enough information for a proper diagnosis and therapeutic management with no need for further imaging [1].

Contraindications and adverse effects of

\section{ultrasound}

The concern about potential harmful side-effects of US is in obstetric examinations. US has limited adverse effects and can be safely used to monitor pregnancy or to investigate the baby under most circumstances [2]. But some precautions have to be taken because, even if US is considered to be a safe imaging technique, it has potential to produce side-effects on the body. These effects are divided between thermal with heating of tissues and mechanical with the formation of small pockets of gas in body fluids or tissues (cavitation). The long term consequences of these effects are still unknown [3]. The American Institute of Ultrasound in Medicine (AIUM) has advocated prudent use of US imaging in pregnancy. The use of US during pregnancy for non-medical purposes such as obtaining fetal videos has been discouraged. Some precautions have to be taken in obstetric US: particularly in early pregnancy exposure time has to be limited to the minimum and thermal and mechanical indices have to be kept as low as possible. Doppler US examination should be avoided when possible in the first trimester of pregnancy $[2,3]$.

\section{Contrast enhanced ultrasound (CEUS)}

US contrast agents (UCA) have been introduced more recently compared to contrast agents in CT and MRI [4]. UCA are safe with only minimal risk to patients. UCA are not renally excreted, they do not interact with renal function and, therefore, can be safely administered to patients with renal insufficiency with no risk of contrast related nephropathy or nephrogenic systemic fibrosis $[5,6]$. UCA do not contain iodine. There is no need for renal and thyroid function tests or any blood assessments prior to UCA injection. There are no anaphylactic reactions described after the usage of UCA but still UCA have a very low rate of pseudoanaphylactoid reactions $(<0.015 \%)$ [4-6], lower than the rate with iodinated contrast agents (0.035-0.095\%). Pseudoanaphylaxy does not involve an allergic reaction but is due to direct mass cell degranulation. Typical side effects are similar to placebo, headache $(2.1 \%)$, nausea $(0.9 \%)$, chest pain $(0.8 \%)$ and chest discomfort $(0.5 \%)[5,6]$ with other side effects with a frequency of $<0.5 \%$. The extravascular administration of UCA has resulted mainly in catheter related adverse events.

Recently the Food and Drug Administration (FDA) in the United States of America (USA) has approved the use of Lumason ${ }^{\mathrm{TM}}$ (marketed as SonoVue ${ }^{\mathrm{TM}}$ Bracco, Milan, outside the USA) for paediatric liver imaging [7].

\section{Pseudoallergies to contrast media administration}

- complement activation-related pseudoallergy

(CARPA)

CARPA is a very rare, but severe, pseudoanaphylactoid reaction after UCA administration, secondary to a relative recently described variant of type 1 hypersensitivity reaction [8]. In contrast to the more common food and drug allergies, CARPAs are not Ig-E mediated and no previous exposure is needed for the allergic reaction to develop [9]. Their incidence is, as mentioned above, very low $(<0.015 \%)$. The amplitude of CARPA reactions may vary from mild (sneezing, tingling, urticaria, pruritus) to severe (wheezing, angioedema, cyanosis and anaphylactic shock). These rare allergic reactions are the only risk associated with UCA [9]. The overall rate of fatalities related to the administration of Sonovue is extremely low $(0.0006 \%)$, clearly lower than the rate of fatalities attributable to iodinated contrast media $(0.001 \%)$ [5].

\section{Computed tomography}

\section{Contraindications and adverse effects}

There are no absolute contraindications for CT examination, although there is still a patient risk due to radiation exposure and contrast media administration.

\section{Ionizing radiation in abdominal imaging}

Abdomino-pelvic CT is an examination with a relatively high-radiation dose in comparison with chest and head CT, due to the large number of radiosensitive organs in the field of view [1]. Abdominal and pelvic CT imparts a dose of $6-10 \mathrm{mSv}$ to the adult patient [10].

Radiologists and radiology staff have a responsibility to keep the radiation dose and exposure to the patient "as low as reasonably achievable" (ALARA), while still obtaining interpretable images allowing an appropriate diagnosis [11]. Every time when a procedure using ionizing radiation can be substituted with a non-ionizing procedure allowing the same amount of information to be obtained and having the same diagnostic benefit, the non-irradiant procedure should be used. Precautions have to be taken, particularly in children and female patients, 
who are more susceptible to the adverse effects of ionizing radiation; children may be ten times more radiosensitive than adults [12]. The relative risk of carcinogenesis is 3 times higher in children below the age of 10 years than it is in adults [13]. If the requirement for an abdominal CT is unavoidable for a correct diagnosis, some simple measures that help to lower the dose should be considered. The patient should be correctly centered within the gantry. A $30-\mathrm{mm}$ off-center distance increases the dose by $12-18 \%$, while a 60 -mm off-center distance increases the dose by $41-49 \%$ [14]. The patient's arms should be raised above the head during the exposure. It was shown that patients with one hand remaining parallel or above the abdomen during a procedure had an increase in dose of about $18 \%$, while patients with both hands remaining above the abdomen had an increase in dose of about $45 \%$ [15]. Non-necessary radiopaque objects should be removed from patients (e.g. wires or leads), as they can increase radiation dose. Many low-radiation dose protocols have been developed during recent years, although most of them also lead to reduced image quality and conspicuity $[16,17]$. Such protocols are particularly useful in younger patients under the age of 40 , or in conditions in which fine detail is not needed, such as in nephrolithiasis [1]. Guidelines such as the American College of Radiology (ACR) Appropriateness Criteria should be used to choose the most appropriate imaging procedure and reduce unnecessary irradiation [1].

\section{Contrast media in CT scanning}

Contrast media administration is particularly useful in abdominal pathology. In practice most of the indications for abdominal CT need contrast media administration. Exceptions are scans for detection and evaluation of urolithiasis and scans for depiction of pneumoperitoneum in suspected visceral perforation $[18,19]$.

\section{Allergies to contrast media administration}

Allergy is the main pitfall of CT-contrast media administration. Allergies to contrast media are rare with only $0.6 \%$ of patients developing allergic reactions. Severe allergic reactions have an even lower incidence, occurring in $0.04 \%$ of patients [20]. Nearly all life-threatening reactions to contrast media occur in the first 20 minutes after contrast media injection [1].

A history of a prior allergy to iodinated contrast media is considered a strong relative contraindication to contrast media administration. Other known allergies, including allergies to Gadolinium (Gd)-based contrast media are not considered contraindications for the administration of iodinated contrast media [21]. However, patients with unrelated allergies have a 2-3 fold higher risk of developing allergic reactions to contrast media [22]. Patients with prior allergy to contrast media have a 5-times greater risk of developing an allergic reaction when contrast media is injected a second time. The use of non-ionic contrast media has contributed to a significant decrease in adverse reactions during contrast media administration [23]. Infants and elderly patients have a lower risk of developing allergic reactions to contrast media administration than middle-aged people.

If contrast media injection is essential for diagnostic purposes, it can be performed even in patients known with previous allergies to iodinated contrast media, after premedication with corticosteroids and antihistamine drugs. Changing the contrast medium has also lowered the incidence of repeated allergic reactions [24].

The pretesting of patients to contrast media does not predict which patients are prone to allergic reactions and does not lower the incidence of contrast media reactions. In a meta-analysis of over 300,000 cases, the predictive value and sensitivity of pretesting for anticipating the reaction to contrast media were extremely low, with values of $1.2 \%$ and $3.7 \%$ respectively [25].

\section{Contrast media use in hyperthyroidism}

In patients with symptomatic hyperthyroidism the administration of contrast media is contraindicated because of the risks of developing thyrotoxicosis [21]. Patients with Graves disease and patients with thyroid goiter are at increased risk of developing thyrotoxicosis. The risk is significantly higher in areas with iodine deficiency. Iodine induced thyrotoxicosis is more frequent among elderly and side-effects of iodine administration are more severe in patients with an associated cardiovascular risk [22]. All at-risk patients should be monitored after contrast media administration, preferably by endocrinologists. Selected patients may benefit from prophylactic thyrostatic therapy [26]. Contrast media administration does not interfere with thyroid function in patients with a normally secreting thyroid gland [21].

\section{Contrast induced nephropathy}

Contrast induced nephropathy (CIN) is a term used to describe a sudden deterioration in renal function caused by the intravascular administration of contrast medium. One of the most used criteria for the diagnosis of CIN is an increase of $0.5 \mathrm{mg} / \mathrm{dl}$ over baseline serum creatinine [27]. Iodinated contrast media acts as an independent risk factor for developing CIN in patients with a GFR lower than $30 \mathrm{ml} / \mathrm{min} / 1.73 \mathrm{~m}^{2}$, but is rarely nephrotoxic in patients with GFR values between 30 and $45 \mathrm{ml} / \mathrm{min} / 1.73$ $\mathrm{m}^{2}[28,29]$. Other risk factors for developing CIN such as diabetes mellitus, arterial hypertension, higher doses of contrast media injected and multiple administrations of contrast media have been proposed but there is no clear proof of an association with CIN [30,31]. Each case should be judged on a patient-by-patient basis, and all 
risks, benefits, and alternatives should be taken into account [32]. Patients at high risk for CIN may benefit from hemodialysis after the procedure. Anuric patients in endstage renal disease with no transplanted kidneys are not at risk of developing CIN; contrast media can be safely administered to such patients [32].

\section{Patients undergoing treatment with Metformin or}

Metformin-containing drug combinations

Metformin is not an independent risk factor for developing acute kidney injury or CIN. The risk from metformin is that patients who develop acute kidney injury while undergoing treatment will develop lactic acidosis more quickly and with more prolonged effects. The recommendation is that Metformin should not be stopped for patients with a GFR higher that $60 \mathrm{ml} / \mathrm{min} / 1.73 \mathrm{~m}^{2}$. Administration should be stopped for 48 hours only in patients with impaired renal function [27].

\section{Magnetic resonance imaging}

\section{Absolute contraindications}

Absolute contraindications to MR imaging include the presence of "MRI unsafe" implants or devices, and the presence of foreign bodies, particularly if these are located close to the eyes or major vessels.

\section{Pacemakers and implantable cardioverter defibrillators (ICD)}

It has been estimated that $50-75 \%$ of patients with pacemakers will have a clinical indication for MRI scanning during the lifetime of the device [33]. The prevalence of pacemakers increases markedly with age; a study performed in Denmark found an overall prevalence of pacemakers of 260/100,000 for a population younger than 65 years, and a prevalence of 2600/100,000 for those older than 75 years [34].

There are a variety of mechanisms by which MRI can affect the functioning of a pacemaker or ICD. The potential pitfalls include device dislodgement, programming changes, asynchronous pacing, activation of anti-tachycardia therapies, and inhibition of pacing output [35]. Sudden death has been reported in patients with pacemakers during or shortly after the MRI procedure [36].

The current recommendations are to avoid conducting MRI studies in patients with pacemakers or ICDs, even if there are reports of patients being safely scanned in centers very experienced in both MRI and cardiovascular pathology [37]. Exceptions are presented by patients who have pacemakers marked as "MRI conditional", although it should be noted that most pacemakers and all ICDs are not "MRI conditional" but "MRI unsafe". For MRI to be performed, the entire system (pacemaker and leads) should be marked as "MRI conditional", not only the pulse generator [37]. Exceptions may occur if there is no other diagnostic tool available and there is an important diagnostic and therapeutic benefit for the patient; in such cases MRI may be performed in very experienced centers [33]. It is recommended that in such cases, a low magnetic field strength scanner should be used, as interference between the magnetic field and the pacemaker increases with the strength of the magnetic field.

Precautions need to be taken in these patients: there is a need to continuously monitor consciousness, heart rate, blood pressure, and oxygen saturation. Visual and acoustic contact must be maintained with the patient throughout the procedure, with the patient being instructed to report any unusual sensations or problems. It must be possible to immediately terminate the scan and evacuate the patient from the scanner, with the staff being trained in advanced cardiac life support. After the procedure, the device must be evaluated to check that it is functioning correctly, with reprogramming sometimes being required [38].

MR studies are also to be avoided in patients with retained transvenous pacemakers and defibrillator leads.

\section{Implanted neurostimulator}

Exposure to MRI can lead to heating of tissue, induced voltages in the neurostimulator and lead dislodgement. The FDA (Food and Drug Administration) does not recommend MRI in patients with neurostimulators, even though there are studies that have shown no side effects and no malfunctioning of the devices after MR imaging [39]. However, in the last few years "MRI conditional" neurostimulators have been produced [40].

\section{Metal fragments near the eye or large vessels}

Shifting of metallic foreign bodies under the influence of MRI can lead to damage of vital structures such as nerves, vessels, or the eye. If there is any doubt about the presence or location of a metallic foreign body, an X-ray should be performed prior to the MRI [41]. Surgery to remove the foreign body may be needed to safely perform MRI.

\section{Brain aneurysm clips made from ferromagnetic material}

MRI is not feasible if brain aneurysm clips are made from ferromagnetic materials. If the clips are made from titanium or titanium alloy, the examination is possible. Over the last decade, MRI compatible clips have been used, so the risk of incompatibility is therefore low, although there is a need for this risk to be checked. If the clip has ferromagnetic properties, its displacement by MRI could lead to damage to brain tissue or hemorrhage [42].

There is a strong recommendation that MRI should not be performed until complete documentation regard- 
ing the clips is obtained, and the manufacturers guarantee that the clips are MRI-safe. All intracranial clips manufactured in 1995 or later are considered MR conditional by the manufacturer and can be scanned without further testing. Previous MR imaging of a patient with a brain aneurysm clip cannot be considered indicative of the safe usage of MRI in that patient; data regarding the MR compatibility of the clip are still needed [37].

\section{Hemodynamic monitoring and temporary pacing \\ devices}

Both pulmonary artery monitoring catheters and temporary transvenous pacing leads contain non-ferromagnetic but electrically conductive material; this makes them inappropriate for MR imaging [43]. The radiofrequency pulses generated during an MRI examination may induce currents that could lead to thermal injuries [44].

\section{Hemodynamic support devices}

Devices such as intracardiac or intra-aortic pumps have not been tested with regard to their MRI safety. The fact that they contain ferromagnetic material and electrical components leads to the recommendation that MRI is contraindicated [28].

\section{Contrast media in MRI scanning \\ Gadolinium and the brain}

A number of studies have demonstrated that gadolinium chelates used as contrast media for MR imaging accumulate in brain tissue $[45,46]$. High signal intensity on T1-weighted images in brain regions such as the globus pallidus and nucleus dentatus after repeated administration of Gd-based contrast media correlates with $\mathrm{Gd}$ accumulation in these areas $[47,48]$.

An important problem that rises is whether the type of Gd-based agent affects gadolinium deposition. Depending on the type of ligand and charge, the Gd-based agents are classified as linear ionic, linear non-ionic, macrocyclic ionic and macrocyclic non-ionic [49]. There are a series of retrospective human studies that showed that $\mathrm{T} 1$ signal changes in the dentate nucleus and globus pallidus are higher in patients exposed to linear agents. Moreover, prospective studies conducted using animals reconfirmed that there is an association between exposure to linear Gd-based agents and gadolinium brain deposition, whereas macrocyclic Gd-based agents did not produce significant $\mathrm{T} 1$ signal changes in the brain structures [50]. This phenomenon can be explained by the chemical structure of the contrast media, which causes different thermodynamic and kinetic stability. Linear agents are less stable than the macrocylcic ones and they might release more gadolinium [51].

No studies have yet shown that gadolinium retained in the brain leads to side effects. A recent report from the
FDA, published in May 2017 [52], suggests that no adverse effects from gadolinium retained in the brain have been identified. Still, because of the unknown long-term potential side effects of Gd-based contrast agents, prudence is recommended in their administration, particularly in the pediatric population. Therefore, gadolinium based contrast agents should only be administered when there is an imperious need, and sequences such as diffusion-weighted imaging should be used to replace administration of contrast media [53].

\section{Nephrogenic systemic fibrosis}

Nephrogenic systemic fibrosis (NSF) is a rare lifethreatening disease that affects patients with renal failure who have had Gd-based contrast agents administered. Because of the risk of developing NSF, patients with a glomerular filtration rate (GFR) lower than $30 \mathrm{ml} / \mathrm{min}$ should not receive Gd-based contrast agents. Current evidence suggests that contrast agents may be classified as high risk (gadopentetic acid), medium risk (gadobenic acid), or low risk (gadoteridol) [54]. Recent clinical evidence suggests that the administration of group II Gdbased contrast agents is associated with a very low or possibly non-existent risk of developing NSF [54].

\section{Allergies reactions to Gd-based contrast media}

Incidence of allergies to Gd-based contrast media is low, with a reported prevalence between 0.8 and 2.4\% [54]. Most of the adverse effects reported are mild. Only patients with previous allergic reactions to contrast media are considered to be at risk for developing acute adverse reactions after administration of Gd-based contrast media.

\section{Imaging in pregnant women}

Imaging pregnant women remains a controversial issue as exposure to ionizing radiation or contrast media can be associated with fetal risk and unnecessary avoidance of diagnostic tests can lead to non-diagnosis and worsening of disease. Even if the usage of CT inflicts a much lower dose than the exposure associated with fetal harm, their usage is traditionally withheld in pregnant woman.

It is well known that exposure to radiation, accidental or for medical purposes, may have detrimental effects on the embryo and fetus. The most important effects on the fetus are more likely if irradiation is performed between 3 and 17 weeks of gestation, with an irradiation dose higher than $50 \mathrm{mGy}$, and these include malformations, spontaneous abortion, and a diminished IQ and mental retardation [55]. The main determinant of the potential teratogenic effects to the embryo is the radiation dose to the conceptus. The estimated conceptus dose is highly 
variable, ranging from close to $0 \mathrm{mGy}$ in head $\mathrm{CT}$, to 25 mGy in abdomino-pelvic CT, or even 34 mGy in CTaortography [55]. Currently, the Association of American Obstetricians and Gynecologists states that exposure to a single diagnostic procedure with an estimated dose to the conceptus of lower that $50 \mathrm{mGy}$ is not associated with fetal abnormalities or loss of pregnancy [56]. Scanning the head or extremities of the woman has virtually no effect on the fetus. CT pulmonary angiography, a vital diagnostic method in suspected pulmonary embolism, can also be safely achieved, because irradiation to the fetus is low [57]. Abdominal and pelvic CT is recommended in pregnant patients only in situations in which a correct diagnosis is of vital importance. Such situations include severe maternal trauma or urolithiasis complicated by unrelenting pain or fever, and where non-ionizing imaging techniques such as MRI or US have not led to a diagnosis [58]. A single-shot acquisition technique is recommended in order to lower the radiation dose. Women who undergo an imaging procedure with ionizing radiation and then discover that they are pregnant after the procedure need to be appropriately counseled. In the vast majority of such cases, the risks of fetal malformations or childhood cancer are very low. The radiation dose to the conceptus should be calculated and the potential effects correlated with the age of gestation. Therapeutic abortion is very rarely indicated.

In considering available data and the risk of teratogenicity, the ACR concludes that unenhanced MRI is safe during pregnancy, for both foetus and mother, and that no special considerations are recommended for the first (or any other) trimester of pregnancy [37]. But still gadolinium contrast agents should be used for pregnant women only in situations when it significantly improves fetal or maternal outcome [37].

Even if US is routinely used in obstetrics, no data on risks and benefits of CEUS in pregnancies have been made available until now. Animal studies showed no harmful effects with respect to the embryonal, foetal and postnatal development. As a precautionary measure the usage of Sonovue in pregnancies should be avoided [59]. In the literature there are reports of the off-label usage of Sonovue in diagnosing placental or foetal pathology. Worthy of noting is that, during the procedure described, no contrast bubbles were seen in the fetal umbilical circulation [60].

\section{Conclusion}

Imaging is an indispensable tool in the diagnostic work-up of patients with abdominal pathology. There are numerous indications for each imaging technique and, when properly used, each method can offer crucial information for the diagnosis. US has virtually no contraindications and potential harmful effects of the technique are still a subject of debate. The main disadvantage of CT is exposure to ionizing radiation. Contrast administration in CT can be limited by the presence of allergies and the development of CIN in patients with impaired renal function. MRI has over $\mathrm{CT}$ the advantage of not exposing the patient to radiation. Contraindications to MRI are the presence of metallic foreign bodies or MRI "unsafe" implants or devices.

\section{Acknowledgement}

The authors of this paper would like to thank Professor Anthony Rudd from the Faculty of Life Sciences \& Medicine Kings College London for his expert advice and for his availability to revise and improve the paper.

\section{Conflict of interest: none}

\section{References}

1. Caraiani C, Dong Y, Rudd AG, Dietrich CF. Reasons for inadequate or incomplete imaging techniques. Med Ultrason 2018;20:498-507.

2. Abramowicz JS. Benefits and risks of ultrasound in pregnancy. Semin Perinat 2013;37:295-300.

3. Ter Haar G. Ultrasonic imaging: safety considerations. Interface Focus 2011;1:686-697.

4. Ignee A, Atkinson NS, Schuessler G, Dietrich CF. Ultrasound contrast agents. Endosc Ultrasound 2016;5:355 -362 .

5. Sidhu PS, Cantisani V, Dietrich CF, et al. The EFSUMB Guidelines and Recommendations for the Clinical Practice of Contrast-Enhanced Ultrasound (CEUS) in Non-Hepatic Applications: Update 2017 (Short Version). Ultraschall Med 2018;39:154-180.

6. Sidhu PS, Cantisani V, Dietrich CF, et al. The EFSUMB Guidelines and Recommendations for the Clinical Practice of Contrast-Enhanced Ultrasound (CEUS) in Non-Hepatic Applications: Update 2017 (Long Version). Ultraschall Med 2018;39:e2-e44.

7. Dietrich CF, Averkiou M, Nielsen MB, et al. How to perform Contrast-Enhanced Ultrasound (CEUS). Ultrasound Int Open 2018;4:E2-E15.

8. Szebeni J. Complement activation-related pseudoallergy: a new class of drug-induced acute immune toxicity. Toxicology 2005;216:106-121.

9. Muskula PR, Main ML. Safety With Echocardiographic Contrast Agents. Circ Cardiovasc Imaging 2017;10. doi:10.1161/CIRCIMAGING.116.005459.

10. Tsapaki V, Rehani M, Saini S. Radiation safety in abdominal computed tomography. Semin Ultrasound CT MR 2010;31:29-38. 
11. Mettler FA Jr, Huda W, Yoshizumi TT, Mahesh M. Effective doses in radiology and diagnostic nuclear medicine: a catalog. Radiology 2008;248:254-263.

12. Strauss KJ, Kaste SC. The ALARA (as low as reasonably achievable) concept in pediatric interventional and fluoroscopic imaging: striving to keep radiation doses as low as possible during fluoroscopy of pediatric patients--a white paper executive summary. Radiology 2006;240:621622.

13. Hall EJ. Radiation biology for pediatric radiologists. Pediatr Radiol 2009;39 Suppl 1:S57-S64.

14. Li J, Udayasankar UK, Toth TL, Seamans J, Small WC, Kalra MK. Automatic patient centering for MDCT: effect on radiation dose. AJR Am J Roentgenol 2007;188:547552.

15. Brink M, de Lange F, Oostveen LJ, et al. Arm raising at exposure-controlled multidetector trauma CT of thoracoabdominal region: higher image quality, lower radiation dose. Radiology 2008;249:661-670.

16. Boedeker KL, McNitt-Gray MF. Application of the noise power spectrum in modern diagnostic MDCT: part II. Noise power spectra and signal to noise. Phys Med Biol 2007;52:4047-4061.

17. Huda W, Ogden KM, Samei E, et al. Reconstruction filters and contrast detail curves in CT. Proc SPIE 6917, Medical Imaging 2008: Image Perception, Observer Performance, and Technology Assessment. 2008;691710.

18. Niemann T, Kollmann T, Bongartz G. Diagnostic performance of low-dose CT for the detection of urolithiasis: a meta-analysis. AJR Am J Roentgenol 2008;191:396-401.

19. Stapakis JC, Thickman D. Diagnosis of pneumoperitoneum: abdominal CT vs. upright chest film. J Comput Assist Tomogr 1992;16:713-716.

20. Wang CL, Cohan RH, Ellis JH, Caoili EM, Wang G, Francis IR. Frequency, outcome, and appropriateness of treatment of nonionic iodinated contrast media reactions. AJR Am J Roentgenol 2008;191:409-415.

21. van der Molen AJ, Thomsen HS, Morcos SK; Contrast Media Safety Committee, European Society of Urogenital Radiology (ESUR). Effect of iodinated contrast media on thyroid function in adults. Eur Radiol 2004;14:902-907.

22. Dunn JT, Semigran MJ, Delange F. The prevention and management of iodine-induced hyperthyroidism and its cardiac features. Thyroid 1998;8:101-106.

23. Katayama H, Yamaguchi K, Kozuka T, Takashima T, Seez $\mathrm{P}$, Matsuura K. Adverse reactions to ionic and nonionic contrast media. A report from the Japanese Committee on the Safety of Contrast Media. Radiology 1990;175:621-628.

24. Abe S, Fukuda H, Tobe K, Ibukuro K. Protective effect against repeat adverse reactions to iodinated contrast medium: Premedication vs. changing the contrast medium. Eur Radiol 2016;26:2148-2154.

25. Yamaguchi K, Katayama H, Takashima T, Kozuka T, Seez P, Matsuura K. Prediction of severe adverse reactions to ionic and nonionic contrast media in Japan: evaluation of pretesting. A report from the Japanese Committee on the Safety of Contrast Media. Radiology 1991;178:363-367.
26. Nygaard B, Nygaard T, Jensen LI, et al. Iohexol: effects on uptake of radioactive iodine in the thyroid and on thyroid function. Acad Radiol 1998;5:409-414.

27. Baerlocher MO, Asch M, Myers A. Five things to know about...metformin and intravenous contrast. CMAJ 2013;185:E78.

28. Davenport MS, Khalatbari S, Cohan RH, Dillman JR, Myles JD, Ellis JH. Contrast material-induced nephrotoxicity and intravenous low-osmolality iodinated contrast material: risk stratification by using estimated glomerular filtration rate. Radiology 2013;268:719-728.

29. McDonald JS, McDonald RJ, Carter RE, Katzberg RW, Kallmes DF, Williamson EE. Risk of intravenous contrast material-mediated acute kidney injury: a propensity scorematched study stratified by baseline-estimated glomerular filtration rate. Radiology 2014;271:65-73.

30. Parfrey PS, Griffiths SM, Barrett BJ, et al. Contrast material-induced renal failure in patients with diabetes mellitus, renal insufficiency, or both. A prospective controlled study. N Engl J Med 1989;320:143-149.

31. Abujudeh HH, Gee MS, Kaewlai R. In emergency situations, should serum creatinine be checked in all patients before performing second contrast CT examinations within 24 hours? J Am Coll Radiol 2009;6:268-273.

32. Katzberg RW, Newhouse JH. Intravenous contrast medium-induced nephrotoxicity: is the medical risk really as great as we have come to believe? Radiology 2010;256:2128.

33. Faris OP, Shein M. Food and Drug Administration perspective: Magnetic resonance imaging of pacemaker and implantable cardioverter-defibrillator patients. Circulation 2006; 114:1232-1233.

34. Andersen C, Green A, Madsen GM, Arnsbo P. The epidemiology of pacemaker implantations in Fyn county, Denmark. Pacing Clin Electrophysiol 1991;14:1614-1621.

35. Levine GN, Gomes AS, Arai AE, et al. Safety of magnetic resonance imaging in patients with cardiovascular devices: an American Heart Association scientific statement from the Committee on Diagnostic and Interventional Cardiac Catheterization, Council on Clinical Cardiology, and the Council on Cardiovascular Radiology and Intervention: endorsed by the American College of Cardiology Foundation, the North American Society for Cardiac Imaging, and the Society for Cardiovascular Magnetic Resonance. Circulation 2007; 116:2878-2891.

36. Ferris NJ, Kavnoudias H, Thiel C, Stuckey S. The 2005 Australian MRI safety survey. AJR Am J Roentgenol 2007;188:1388-1394.

37. Expert Panel on MR Safety, Kanal E, Barkovich AJ, et al. ACR guidance document on MR safe practices: 2013. J Magn Reson Imaging 2013;37:501-530.

38. Shinbane JS, Colletti PM, Shellock FG. MR in patients with pacemakers and ICDs: Defining the issues. J Cardiovasc Magn Reson 2007;9:5-13.

39. Elkelini MS, Hassouna MM. Safety of MRI at 1.5Tesla in patients with implanted sacral nerve neurostimulator. Eur Urol 2006;50:311-316. 
40. De Andres J, Martinez-Sanjuan V, Fabregat-Cid G, Asensio-Samper JM, Sanchis-Lopez N, Villanueva-Perez V. MRI-compatible spinal cord stimulator device and related changes in patient safety and imaging artifacts. Pain Med 2014;15:1815-1819.

41. Kanal E, Barkovich AJ, Bell C, et al. ACR guidance document for safe MR practices: 2007. AJR Am J Roentgenol 2007;188:1447-1474.

42. Kanal E, Shellock FG. Aneurysm clips: effects of long-term and multiple exposures to a 1.5-T MR system. Radiology 1999;210:563-565.

43. Dill T. Contraindications to magnetic resonance imaging: non-invasive imaging. Heart 2008;94:943-948.

44. Dempsey MF, Condon B. Thermal injuries associated with MRI. Clin Radiol 2001;56:457-465.

45. Kanda T, Osawa M, Oba H, et al. High Signal Intensity in Dentate Nucleus on Unenhanced T1-weighted MR Images: Association with Linear versus Macrocyclic Gadolinium Chelate Administration. Radiology 2015;275:803-809.

46. McDonald RJ, McDonald JS, Kallmes DF, et al. Intracranial Gadolinium Deposition after Contrast-enhanced MR Imaging. Radiology 2015;275:772-782.

47. Quattrocchi CC, Mallio CA, Errante Y, Beomonte Zobel B. High T1 Signal Intensity in Dentate Nucleus after Multiple Injections of Linear Gadolinium Chelates. Radiology 2015;276:616-617.

48. Quattrocchi CC, Mallio CA, Errante Y, et al. Gadodiamide and Dentate Nucleus T1 Hyperintensity in Patients With Meningioma Evaluated by Multiple Follow-Up ContrastEnhanced Magnetic Resonance Examinations With No Systemic Interval Therapy. Invest Radiol 2015;50:470-472.

49. Frenzel T, Lengsfeld P, Schirmer H, Hutter J, Weinmann HJ. Stability of gadolinium-based magnetic resonance imaging contrast agents in human serum at 37 degrees C. Invest Radiol 2008;43:817-828.

50. Guo BJ, Yang ZL, Zhang LJ. Gadolinium Deposition in Brain: Current Scientific Evidence and Future Perspectives. Front Mol Neurosci 2018;11:335.

51. Radbruch A, Haase R, Kieslich PJ, et al. No Signal Intensity Increase in the Dentate Nucleus on Unenhanced T1- weighted MR Images after More than 20 Serial Injections of Macrocyclic Gadolinium-based Contrast Agents. Radiology 2017;282:699-707.

52. FDA identifies no harmful effects to date with brain retention of gadolinium-based contrast agents for MRIs; review to continue. Available at: https:/www.fda. gov/files/drugs/published/FDA-identifies-no-harmfuleffects-to-date-with-brain-retention-of-gadoliniumbased-contrast-agents-for-MRIs--review-to-continue. pdf.

53. Kanal E, Tweedle MF. Residual or retained gadolinium: practical implications for radiologists and our patients. Radiology 2015;275:630-634.

54. Association of Anaesthetists of Great B, Ireland, Farling PA, Flynn PA, et al. Safety in magnetic resonance units: an update. Anaesthesia 2010;65:766-770.

55. McCollough CH, Schueler BA, Atwell TD, et al. Radiation exposure and pregnancy: when should we be concerned? Radiographics 2007;27:909-917.

56. ACOG Committee on Obstetric Practice. ACOG Committee Opinion. Number 299, September 2004 (replaces No. 158, September 1995). Guidelines for diagnostic imaging during pregnancy. Obstet Gynecol 2004;104:647651 .

57. Schuster ME, Fishman JE, Copeland JF, Hatabu H, Boiselle PM. Pulmonary embolism in pregnant patients: a survey of practices and policies for CT pulmonary angiography. AJR Am J Roentgenol. 2003;181:1495-1498.

58. Lowdermilk C, Gavant ML, Qaisi W, West OC, Goldman SM. Screening helical CT for evaluation of blunt traumatic injury in the pregnant patient. Radiographics 1999;19 Spec No:S243-S255.

59. European Medicines Agency (EMA). Sonovue epar product information. Available at: https://www.ema.europa.eu/ en/documents/product-information/sonovue-epar-productinformation_en.pdf.

60. Windrim R, Kingdom J, Jang HJ, Burns PN. Contrast enhanced ultrasound (CEUS) in the prenatal evaluation of suspected invasive placenta percreta. J Obstet Gynaecol Can2016;38:975-978. 